\title{
Did the 20I I AAP recommendations on youth HIV testing change practice? Trends from a large urban adolescent program
}

This article was published in the following Dove Press journal:

HIVIAIDS - Research and Palliative Care

26 April 2017

Number of times this article has been viewed

\section{Sujatha Seetharaman' Cathryn L Samples ${ }^{2}$ \\ Maria Trent ${ }^{3}$ \\ 'Division of Adolescent Medicine, Stanford University Medical Center, Palo Alto, CA, ${ }^{2}$ Division of Adolescent Medicine, Boston Children's Hospital, Harvard Medical School, Boston, MA, ${ }^{3}$ Division of General Pediatrics and Adolescent Medicine, Johns Hopkins Bloomberg School of Public Health, Johns Hopkins School of Medicine, Baltimore, MD, USA}

Correspondence: Sujatha Seetharaman Division of Adolescent Medicine, Stanford University Medical Center, 770 Welch Road, Palo Alto, CA 94304, USA

Email sujathas@stanford.edu
Purpose: The purpose of this study was to determine whether there is adherence to the October 2011 American Academy of Pediatrics (AAP) recommendations for HIV screening in a large urban adolescent program with availability of a publicly funded program providing free, confidential, sexually transmitted infection (STI) and HIV counseling and testing (then rapid or third generation HIV testing), nested in the same adolescent clinic.

Methods: We conducted a retrospective chart review of HIV screening trends among 13- to 24-year-old patients tested for HIV during periods of January 2010 to June 2011 (18 months pre-AAP recommendations period) and July 2011 to December 2012 (18-month period, which included 15 months after the AAP recommendations).

Results: During the period of January 2010 to June 2011, there were 22 tests/1,000 medical visits ( $\mathrm{N}=824$ of 37,520 medical visits), and during the period of July 2011 to December 2012, there were 27 tests $/ 1,000$ medical visits $(\mathrm{N}=1,068$ of 38,763 medical visits $)(p<0.0001$, odds ratio [OR] 1.26). The number of 13- to 18-year-old patients screened in the pre-AAP period was 150 , compared to 297 in the second 18 -month period $\left(X^{2}=43.3, d f=1, p<0.0001\right)$. A summative risk profile score of 0-9 was created in the form of a continuous variable, with a risk score of 0 for those with no risk factor identified and 1 point for each risk behavior identified. The proportion of HIV test clients with zero-specified risk (a risk score of "0") increased from 2010 to 2012.

Conclusion: Release of the 2011 AAP HIV testing guidelines was associated with a modest increase in HIV screening and a shift toward testing younger people and away from risk-based screening.

Keywords: adolescents, HIV screening, 2011 AAP HIV recommendations, risk-based HIV screening, HIV risk factors

\section{Introduction}

HIV disproportionately affects adolescents and young adults compared with the overall population. ${ }^{1}$ Despite advances in treatment, HIV is one of the top 10 causes of death in the 20- to 24-year age group. ${ }^{2}$ The Centers for Disease Control and Prevention (CDC) estimates that in the year 2010, individuals aged 13 to 24 years accounted for $26 \%$ of estimated new HIV infections. ${ }^{2,3}$ Among adolescents and young adults living with HIV infection, 44\% were undiagnosed, the highest percentage of any age group. ${ }^{3}$ Early treatment with antiretroviral therapy helps in maintaining a high CD4 count, thus helping in immunologic recovery and decreasing HIV-associated morbidity, mortality, and transmission. ${ }^{4,5}$ Without HIV screening, adolescents and 
young adults cannot take advantage of HIV treatment that can promote health and reduce the risk of transmission to others. We have witnessed a $20 \%$ decline in the global HIV transmission rates between 2001 and 2007 due to better prevention and treatment efforts. ${ }^{6}$

In 2013, the United States Preventive Services Task Force (USPSTF) recommended "routine HIV screening" for all people between 15 and 64 years of age and for all older and younger persons at risk, as a grade " $\mathrm{A}$ " recommendation (grade "A" recommendation means that the USPSTF recommends the service, and there is high certainty that the net benefit is substantial). ${ }^{7}$ Previously, the USPSTF had endorsed a "risk-based HIV screening" approach for adolescents, in part due to concerns that routine HIV testing could cause psychological harm and that treatment could have serious side effects. The change in the USPSTF recommendation reflects a growing support for routine HIV screening ${ }^{7}$ and recognition of the fact that individuals who have the infection, and are unaware of their status, contribute to the public health problem of HIV. The 2013 USPSTF recommendation is consistent with the 2006 CDC's recommendation ${ }^{8}$ for, 1) voluntary opt-out testing for all patients aged 13-64 years, unless HIV prevalence is $<0.1 \%, 2$ ) annual screening of all patients at high risk (with tuberculosis, sexually transmitted infection [STI]) for HIV infection, and 3) elimination of separately written consent for HIV testing.

In October 2011, the American Academy of Pediatrics (AAP) recommended that HIV screening be performed at least once among adolescents aged 16-18 years (or sooner, if at risk at an earlier age) and then yearly if a youth is at recurring risk ${ }^{9}$ (Table $\mathrm{S} 1$ ). The AAP also recommends that the consent of the adolescent should be sufficient to provide testing and treatment for HIV infection or STIs. ${ }^{9}$ While this appears to be complementary to the 2006 CDC guidelines as well as the later 2013 USPSTF guidelines for HIV testing, the CDC reported no evidence of increases in self-reported HIV testing among high school students during 2005-2011 or among young adults aged 18-24 years during 2000-2010. ${ }^{10}$ A prior AAP survey suggested that only $50 \%$ of pediatricians recommended that STI screening be performed for all sexually active youths and only $28 \%$ recommended youth HIV testing. ${ }^{11}$ The aim of our study was to assess how well the October 2011 AAP HIV screening recommendations had been incorporated into clinical practice in a large urban adolescent medicine program serving teens and young adults. We hypothesized that after the recommendations, more HIV testing would be done, and that younger and low-risk youth would be screened.

\section{Methods}

\section{Sample}

Retrospective quarterly data for HIV testing among 13- to 24-year-old patients during periods of January 2010 to June 2011 (18 months pre-AAP recommendations period) and July 2011 to December 2012 (18-month period, which included 15 months after the AAP recommendations) were collected from a large urban adolescent medicine program in the northeast, providing primary and subspecialty care to more than 4,000 youth between 10 and 25 years of age, with approximately 15,000 clinic visits annually. This program also offered HIV testing on site through a publicly funded program providing free, confidential, STI and HIV counseling and rapid or third generation HIV testing, nested in the same adolescent clinic.

\section{Measures}

Demographic measures (race/ethnicity, gender, and age), HIV testing data, and sexual behavior risk factors (e.g., unprotected intercourse, history of STIs, intravenous drug use [IDU], and history of survival sex) for patients in both the adolescent clinic practice and the publicly funded program were reviewed retrospectively. Data were deidentified at the time of data extraction and database development. Deidentified program data, which included risk and demographic variables, were used to describe HIV testing ordered through the publicly funded program. Boston Children's Hospital Institutional Review Board approved the retrospective data analysis. To describe risk consistently, a summative risk profile score between 0 and 9 was created in the form of a continuous variable (Table 1). Each risk behavior was given 1 point. Data from patients who were, 1) $<13$ and $>24$ years of age, 2) HIV testing done as part of sexual assault or postexposure prophylaxis follow-up, and 3) those with missing data, were excluded.

Table I Risk behaviors used to calculate the risk profile score

\begin{tabular}{ll}
\hline Risk behavior & Score \\
\hline Unprotected sex & $\mathrm{I}$ \\
Sex with HIV+ person & $\mathrm{I}$ \\
Past history of a STI & $\mathrm{I}$ \\
MSM & $\mathrm{I}$ \\
Bisexual partners & $\mathrm{I}$ \\
Sex with IDU+ person & $\mathrm{I}$ \\
Sex with STI+ person & $\mathrm{I}$ \\
History of survival sex & $\mathrm{I}$ \\
History of IDU & $\mathrm{I}$ \\
No risk factor identified & 0 \\
\hline
\end{tabular}

Abbreviations: STI, sexually transmitted infection; MSM, men having sex with men; IDU, intravenous drug use. 


\section{Analysis}

Descriptive and bivariate exploratory analyses were performed as follows:

1) To examine trends in the number of HIV tests done, the total number of tests from both programs was added and examined for changes before and after the AAP recommendations during the two periods. It was also compared with the estimated total number of primary care visits for the two periods, based on historical data. Due to the unavailability of the exact number of primary care visits for each year in the study period, the exact rate of testing could not be calculated. Chi-square test was performed to compare the difference in the proportion of HIV screening in the two periods.

2) To examine trends in risk profile of test clients, a two sample $z$-test of proportions was used to compare the risk profile of test clients in the adolescent clinic and the public funded programs from the years 2010 to 2012 .

\section{Ethics/copyright information}

The Boston Children's Hospital Institutional Review Board approved the study, and a waiver of informed consent was granted for review of medical records for quality improvement purposes.

\section{Results}

Selected demographics of the test clients by year are presented in Table 2 . Test clients were predominately non-White multiracial youth, females, and young adults, which was different from the clinic population that had 36\% White, 32\% African American, 14\% Hispanic, 2\% Asian, 3\% other, and $13 \%$ unknown. In the 18 -month comparison, the number of HIV tests and the number and proportion of younger clients tested increased (Table 3). A ratio of HIV tests to overall clinic visits was assessed to determine the HIV screening prevalence for each period. The number of eligible HIV

Table 2 Gender and race of clients who were tested for HIV, by year

\begin{tabular}{llll}
\hline Variable & $\mathbf{2 0 1 0}$ & $\mathbf{2 0 I ~ I ~}$ & $\mathbf{2 0 I 2}$ \\
& $(\mathbf{N}=\mathbf{6 3 6})$ & $\mathbf{( N = 8 0 2 )}$ & $\mathbf{( N = 6 6 5 )}$ \\
\hline Females & $64 \%$ & $61 \%$ & $66 \%$ \\
Males & $36 \%$ & $39 \%$ & $34 \%$ \\
African American & $47 \%$ & $50 \%$ & $52 \%$ \\
Asian & $3 \%$ & $5 \%$ & $4 \%$ \\
White & $25 \%$ & $20 \%$ & $24 \%$ \\
Other & $25 \%$ & $25 \%$ & $19 \%$ \\
\hline
\end{tabular}

tests performed increased in the post-AAP period by $30 \%$. During the period of January 2010 to June 2011, there were 22 tests $/ 1,000$ medical visits $(\mathrm{N}=824$ of 37,520 medical visits) and during the period of July 2011 to December 2012, there were 27 tests $/ 1,000$ medical visits $(\mathrm{N}=1,068$ of 38,763 medical visits), with the difference being statistically significant $\left(X^{2}=24.4, d f=1, p<0.0001\right)$ (Figure 1 ). Patients were $26 \%$ more likely to have HIV testing during the period of July 2011 to December 2012 compared to the pre-AAP period (odds ratio $[\mathrm{OR}]=1.26,95 \% \mathrm{CI} 1.15-1.38$, $p<0.0001)$. The number of 13-18-year-old patients screened in the pre-AAP period was 150, compared to 297 during the period of July 2011 to December 2012, which was 15 months during and post-AAP recommendation period $\left(X^{2}=43.3\right.$, $d f=1, p<0.0001)$.

The proportion of test clients with zero-specified risk (a risk score of "0") did increase from 2010 to 2012. A twosample $z$-test of proportions for provider-ordered test patients and the publicly funded HIV program showed a statistically significant increase in the proportion of patients with a risk score of 0 from 2010 to 2012, in both programs. For the provider-ordered tests in the adolescent clinic, the proportion

Table 3 Age of the test clients and the number of tests during the time period of January 2010 to June $201 \mathrm{I}$ and July $20 \mathrm{II}$ to December 2012

\begin{tabular}{lll}
\hline Variable & $\begin{array}{l}\text { January 20 I O } \\
\text { to June 20 I I }\end{array}$ & $\begin{array}{l}\text { July 20 I I to } \\
\text { Dec 20 I 2 }\end{array}$ \\
\hline I3- to I8-year-olds tested* & 150 & 297 \\
I9- to 24-year-olds tested & 674 & 77 I \\
Total number of HIV tests** & 824 & 1,068 \\
Number of medical visits & 37,520 & 38,763 \\
Number of HIV tests per I,000 visits & 22 & 27 \\
\hline
\end{tabular}

Notes: ${ }^{*} p$-value $<0.0001$. ${ }^{* *} p$-value $<0.0001$.

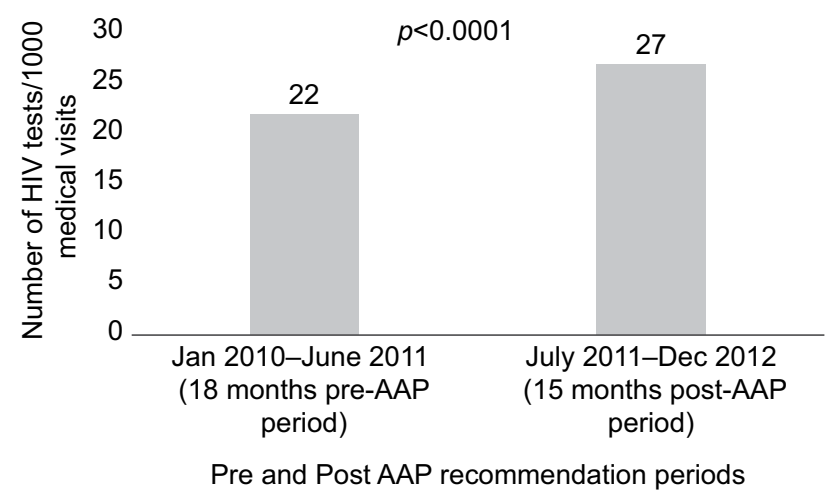

Figure I Number of HIV tests/I,000 medical visits.

Abbreviation: AAP, American Academy of Pediatrics. 
Table 4 Proportion of HIV test clients with a "0" risk score in 2010 and 2012

\begin{tabular}{llll}
\hline Program & $\begin{array}{l}\mathbf{2 0 1 0} \\
\mathbf{( N = 8 0 2 )}\end{array}$ & $\begin{array}{l}\mathbf{2 0 I 2} \\
\mathbf{( N = 6 6 5 )}\end{array}$ & $\begin{array}{l}\text { P-value based } \\
\text { on a two-sample } \\
\text { "z" sample test } \\
\text { of proportions }\end{array}$ \\
\hline $\begin{array}{l}\text { Provider-ordered HIV } \\
\text { tests in the adolescent } \\
\text { clinic }\end{array}$ & $18 \%$ & $37 \%$ & 0.003 \\
$\begin{array}{l}\text { HIV tests ordered } \\
\text { through the publicly } \\
\text { funded program }\end{array}$ & $11 \%$ & $17 \%$ & 0.002 \\
\hline
\end{tabular}

of patients with a risk score of " 0 " increased from $18 \%$ in 2010 to $37 \%$ in $2012(p=0.003)$ (Table 4$)$. For the publicly funded program, the proportion of patients with a risk score of " 0 " increased from $11 \%$ in 2010 to $17 \%$ in 2012 ( $p=$ 0.002) (Table 4).

\section{Discussion}

Our study results suggest that there was a slight increase in the number of HIV tests done after the AAP recommendations, with more HIV screening being done in younger and in low-risk youth after the AAP recommendations. Even with availability of a free confidential HIV testing program nested in the adolescent clinic practice, there was only a modest increase in HIV tests being performed during the 15 months during and post-AAP recommendation period. The shift toward screening younger people and youth with little or no risk could be due to increased provider and youth awareness and due to a shift toward routine HIV screening after the 2011 AAP recommendations. Rapid HIV testing that provides results within 20 minutes and was available at the publicly funded program, has previously been shown greater feasibility and acceptability among the youth compared to the third generation nonrapid enzyme-linked immunosorbent assay and Western blot for HIV infection offered by the adolescent clinic at that time. ${ }^{12}$

Many factors could explain the lack of dramatic response to the recommendations. When this study was planned, Massachusetts had just eliminated requirements for written informed consent for each HIV test ordered by a provider. However, the hospital did not authorize the elimination of a written consent until fall 2013, so the impact of elimination of written consent is not tracked in this paper. Other institutional-level barriers (e.g., insurance, confidentiality) and personal barriers (e.g., time available for counseling visit, needle phobia) also need to be addressed. The modest increase in HIV testing in the post-AAP recommendation period might also be related to not offering HIV testing for patients who do not appear to be at risk in the context of primary care with a parent present or discomfort around sexual health topics though this is unlikely in this urban academic adolescent medicine clinic. Clients may also have received testing elsewhere or declined screening.

The findings of this study should be considered in the light of several general limitations. Due to quarterly availability of data, the comparison was made between the two specified periods of January 2010 to June 2011 and July 2011 to December 2012, which was 3 months during and 15 months after the AAP recommendations were released online. This 15 -month period may be inadequate to examine the impact of recommendations on clinical practice in the absence of a national campaign to enhance use by pediatricians. The delay in implementation of institutional changes to allow verbal consent may also have impacted HIV screening as part of routine care. Data analysis is limited to those who were offered and received HIV testing, limiting the generalizability of the findings. Finally, the reports of risk behaviors were based on self-report and data available in the medical records. Provider documentation of sexual and screening histories varied greatly in the general practice compared with the publicly funded program that used counseling staff who completed standard documentation. This approach may result in an underestimate of risk for patients in general practice.

Further quality improvement and assurance efforts are required to investigate how well the AAP recommendations are being incorporated and to eliminate barriers that still prevent full implementation of routine HIV screening in the care of youth and young adults. Development of strategies to assess both individual youth self-report and medical record documentation of risk and screening practices is warranted. This will increase the proportion of youth aware of their HIV status and facilitate transition of HIV-positive youth into appropriate HIV-related care.

\section{Implications and contribution}

Findings from the study suggest that there has been a slight increase in HIV screening after the 2011 AAP guidelines. Further efforts are required to educate pediatricians and others caring for youth about the 2011 AAP recommendations for HIV screening in adolescents.

\section{Acknowledgments}

We would like to thank Dr. Elaine Isabel Allen and the UCSF CTSI (grant number UL1 TR000004) for their statistical assistance. This project has been funded in part with Federal funds (P20MD000198) from the National Institute of 
Minority Health \& Health Disparities. The content is solely the responsibility of the authors and does not necessarily represent the official views of the National Institutes of Health. An abstract of this paper has been presented as a poster and published in the Journal of Adolescent Health, Volume 56, Issue 2, Supplement 1, Page S52, February 2015.

\section{Disclosure}

The authors report no conflicts of interest in this work.

\section{References}

1. Centers for Disease Control and Prevention. HIV Surveillance in Adolescents and Young Adults. 2011. Available from: http://www.cdc.gov/ hiv/pdf/statistics_surveillance_Adolescents.pdf. Accessed January 12, 2016.

2. Centers for Disease Control and Prevention [webpage on the Internet]. Estimated HIV Incidence in the United States, 2007-2010. HIV Surveillance Supplemental Report 2012. Vol. 17 (No. 4). 2012. Available from: http://www.cdc.gov/hiv/topics/surveillance/resources/ reports/\#supplemental. Accessed February 12, 2016.

3. Centers for Disease Control and Prevention [webpage on the Internet]. Monitoring Selected National HIV Prevention and Care Objectives by Using HIV Surveillance Data - United States and 6 Dependent Areas2013. HIV Surveillance Supplemental Report 2015. Vol. 20 (No. 2). 2015 Available from: http://www.cdc.gov/hiv/library/reports/surveillance/. Accessed February 12, 2016.
4. Lyles RH, Munoz A, Yamashita TE, et al. Natural history of human immunodeficiency virus type 1 viremia after seroconversion and proximal to AIDS in a large cohort of homosexual men. Multicenter AIDS cohort study. J Infect Dis. 2000;181(3):872-880.

5. Marks G, Crepaz N, Senterfitt JW, Janssen RS. Meta-analysis of highrisk sexual behavior in persons aware and unaware they are infected with HIV in the United States: implications for HIV prevention programs. J Acquir Immune Defic Syndr. 2005;39(4):446-453.

6. Holtgrave DR, Hall HI, Prejean J. HIV transmission rates in the United States, 2006-2008. Open AIDS J. 2012;6:26-28.

7. Moyer VA, U.S. Preventive Services Task Force. Screening for HIV: U.S. preventive services task force recommendation statement. Ann Intern Med. 2013;159(1):51-60.

8. Branson BM, Handsfield HH, Lampe MA, et al; Centers for Disease Control and Prevention (CDC). Revised recommendations for HIV testing of adults, adolescents, and pregnant women in health-care settings. MMWR Recomm Rep. 2006;55(RR-14):1-17.

9. Committee on Pediatric AIDS, Emmanuel PJ, Martinez J. Adolescents and HIV infection: the pediatrician's role in promoting routine testing. Pediatrics. 2011;128(5):1023-1029.

10. Centers for Disease Control and Prevention. HIV Testing Trends in the United States, 2000-2011. Atlanta, GA: U.S. Department of Health and Human Services, Centers for Disease Control and Prevention; 2013.

11. Henry-Reid LM, O'Connor KG, Klein JD, Cooper E, Flynn P, Futterman DC. Current pediatrician practices in identifying high-risk behaviors of adolescents. Pediatrics. 2010;125(4):e741-e747.

12. Tuysuzoglu S, Corliss HL, Fitzgerald SM, Abascal BR, Samples CL. Acceptability and feasibility of rapid HIV testing in an adolescent clinic setting: youth testing attitudes, knowledge, and behaviors. J Adolesc Health. 2011;49(6):609-614. 


\section{Supplementary material}

Table SI Brief summary of 20I I key recommendations by the American Academy of Pediatrics on Adolescent and Youth HIV testing

I Screen all adolescents $16-18$ years, at least once, if HIV prevalence $>0.1 \%$

2 If HIV prevalence $<0.1 \%$, screen all sexually active adolescents with identified risk behaviors (e.g., substance, etc.)

3 Perform annual screening for all high-risk youth

$4 \quad$ Routine screening should occur in urgent care and emergency care settings in communities with high HIV prevalence

$5 \quad$ Physicians should be prepared to diagnose acute retroviral syndrome

6 Adolescent consent is sufficient for testing. Parental engagement encouraged. Consider referring to free and confidential community-based testing programs if there are issues with cost or confidentiality

$7 \quad$ Pediatricians need to screen all adolescents for sexual and substance use behaviors using standardized assessments

8 Pediatricians must display tolerance and create an environment for open communication and trust

9 If state law allows, opt-out testing preferred

10 Adolescents with negative testing should receive sexual and substance use risk reduction counseling

II Adolescents with positive tests should be linked to age-appropriate HIV-related health services

12 Pediatricians should advocate for adolescent access to HIV prevention and intervention services

13 Universal coverage, adequate reimbursement, and confidential billing should be core areas of advocacy to ensure optimal preventive services delivery

Note: Data from the American Academy of Pediatrics Policy Statement.'

\section{Reference}

1. Committee on Pediatric AIDS, Emmanuel PJ, Martinez J. Adolescents and HIV infection: the pediatrician's role in promoting routine testing. Pediatrics. 2011;128(5):1023-1029.

\section{Publish your work in this journal}

HIV/AIDS - Research and Palliative Care is an international, peerreviewed open access journal focusing on advances in research in HIV, its clinical progression and management options including antiviral treatment, palliative care and public healthcare policies to control viral spread. The journal is included in PubMed. The manuscript man- agement system is completely online and includes a very quick and fair peer-review system, which is all easy to use. Visit http://www.dovepress. com/testimonials.php to read real quotes from published authors. 